\title{
Carlos Ramírez
}

Escuela de Ciencia Política - Universidad de Artes y Ciencias Sociales

carlos.rev@gmail.com

\section{Cristopher Yáñez-Urbina}

Escuela de Psicología - Universidad de Santiago de Chile

cristopher.urbina@usach.cl

\section{Iván Salinas}

Instituto de Asuntos Públicos - Universidad de Chile

Ivan.salinas@ciae.uchile.cl

\section{Chile, la democracia se acabó: crisis institucional en el 18-O chileno}

\section{Chile, democracy is over: institutional crisis in the chilean 18-O}

\section{Resumen}

El artículo realiza un análisis de los eventos ocurridos entre el 4 de octubre y el 22 de noviembre del 2019 en Chile, con la finalidad tanto de otorgar un panorama político situado al contexto nacional como para poder abstraer aquella disyunción existente entre una política decimonónica basada en la democracia representativa y una conformación social que escapa constantemente de sus esquemas. De tal manera, se aborda tanto el suelo arqueológico desde donde se erige la racionalidad de la democracia representativa, lo que posteriormente es contrastado con las dos principales estrategias desplegadas por la institucionalidad moderna para gestionar el conflicto. Se concluye la existencia de una crisis profunda de la democracia representativa y los mecanismos decimonónicos que queda patente en un actuar que colinda con el fascismo.

Palabras clave: Crisis política, conflicto político, democracia.

\begin{abstract}
The article analyzes the events that occurred between October 4th and November 22nd, 2019 in Chile, with the proposal both to grant a political panorama located in the national context and to be able to abstract that existing function between a nineteenth-century policy based in representative democracy and a social conformation that constantly escapes its schemes. In this way, both the archaeological ground is approached from where the rationality of representative democracy stands. What is subsequently contrasted with the two main strategies deployable by modern institutions to manage the conflict. It concludes the existence of a deep crisis of representative democracy and nineteenth-century mechanisms that is evident in an act that borders fascism.
\end{abstract}

Keywords: Political crises, political conflicts, democracy. 


\section{Introducción}

La democracia en Chile se acabó. Desde mediados de octubre del 2019 se ha hecho patente que yacía muerta desde mucho antes de lo que pudimos percatarnos y que nos encontrábamos habitando el putrefacto cadáver de un orden en descomposición. El fin de la democracia, tal y como la hemos conocido desde hace no más de dos siglos, se ha instalado como un quiebre rotundo de las instituciones decimonónicas, las tecnologías políticas que en ellas pululaban y las formas de gestionar y comprensión lo social.

Con el reciente estallido social que se ha proliferado a lo largo del país, hemos de despedirnos de aquella esquemática de la movilización y la protesta centrada en la representación, a saber: el programa, el partido, la vanguardia y el líder que, como su brazo institucional e instituido, encabeza las demandas; asimismo hemos perdido la organización unificada en la figura heroica, carismática, dura y comprometida con la causa; y se desvanece las consignas claras y delimitadas cuya adhesión permitía trazar un mapa de la ruta a seguir.

En esta línea, la caída de la democracia implica la caída de la movilización tal y como se nos presentaba en la radicalización del Partido Socialista en la década de 1960 (Ortega, 2008), el movimiento de pobladores durante la Unidad Popular (Garcés, 2015), el movimiento universitario en plena dictadura cívico-militar (Toro, 2015) y los movimientos estudiantiles desde la década de 1990 pasando por la revolución pingüina del 2006 hasta el enroque oligárquico de las dirigencias del 2011 (Fleet, 2011; Núñez, 2012; Rifo, 2013), entre tanto otros casos.

El estallido social que ha experimentado Chile carece de una clave de lectura que nos permita no solamente comprender lo que ocurre, sino también desarrollar estrategias políticas para aquel mundo porvenir. En dicho sentido, es necesario circunscribir estas dinámicas en un movimiento mucho más amplio, pero que a la vez se vuelve irreductible a ellos, así encontramos características similares en las manifestaciones ocurridas en Medio Oriente, bautizadas como "Primavera Árabe", en la cual intelectuales como Arditi (2013), desde el postmarxism, hasta Agamben (2017), desde la tradición foucaultiana, han querido delinear el paradigma de la política que viene.

Por lo tanto, en el presente artículo analizamos los eventos ocurridos entre el 4 de octubre y el 22 de noviembre del 2019 en Chile, con la finalidad tanto de otorgar un panorama político 
situado al contexto nacional, como con el objeto de abstraer aquella disyunción existente entre una política decimonónica basada en la democracia representativa y una conformación social que escapa constantemente de sus esquemas. De tal manera, abordamos tres puntos, a saber: primeramente una caracterización de la democracia representativa y el esquema en donde se instala, para luego contrastarlas con las estrategias de gestión del conflicto desplegadas por la institucionalidad; y, finalmente, concluimos con algunas caracterizaciones de las movilizaciones y el panorama que trazan para una política porvenir.

\section{Breve Genealogía de la Democracia Representativa}

La hipótesis que está a la base del presente trabajo, dice relación con la inconmensurabilidad de dos paradigmas políticos en disputa. El nivel de irreductibilidad en ambos extremos es directamente proporcional con la fractura topográfica de los estratos (elementos de saber) y la obsolescencia de las estratégicas (tecnologías de poder) (Deleuze, 1987) que se abre ya entrados en el siglo XXI. Para poder analizar esta fractura y obsolescencia, antes de interrogar el suelo arqueológico y genealógico que ha entrado en crisis en Chile o el tiempo interregno en el que habitamos (Valderrama, 2016), debemos entregar algunas claves de lectura que nos permitan trazar el lento fallecer de la episteme moderna y sus tecnologías de poder.

Entrando en materia, Foucault denomina positividades a la distribución que las palabras y las cosas adoptan en un momento histórico determinado y que constituye su modo de ser, esta distribución es inmediatamente la forma en que ellas son entregadas al saber (Foucault, 1968). Por lo cual, las positividades constituyen el suelo arqueológico de una época dada. Estas positividades entran en relación con lo que Foucault denominará como a priori histórico (Foucault, 1979), siendo estos la "condición de realidad para unos enunciados" (1979; 215-216), es decir, son regímenes de veridicción que habilitan dispersiones, grietas, polémicas, reemplazos y simultaneidades. Son el "zócalo" histórico en la historia como condición de posibilidad de lo enunciable.

Con esto, no podemos reducir el 18-O a un simple agotamiento de legitimidad de las instituciones y su función social, ni la incomprensión o ineptitud de los gobernantes que no se atendrían a los fines racionales del Estado. Estamos hablando de una crisis en el sentido gramsciano del término (Valderrama, 2016), de la episteme moderna y sus tecnologías de poder como a priori histórico, positividad o régimen de veridicción, todos ellos términos encadenados. 
Esto es lo que ha sido fracturado y "obsoletizado" por nuevas formas sociales irreductibles a éstas. Este es el gran drama de nuestra época, de ahí la hipótesis fuerte del fin de la democracia como la conocemos, la cual está anclada a dicha episteme y sus tecnologías.

Considerando que un a priori histórico es la condición de posibilidad de lo enunciable y que las positividades son el tipo de distribución de los seres que constituyen el saber, mientras que ambos articulan regímenes de veridicción, es que la modernidad y sus tecnologías de poder pueden ser abordadas como una crisis de paradigmas políticos, renunciando de entrada a toda estructura-social transhistórica y todo sujeto racional que encarnaría el punto originario y trasendental de la historia.

Siguiendo la descripciones de Foucault (1968), la Modernidad se sitúa a partir del siglo XVIII en el quiebre con la época clásica o la episteme renacentista. Dicho proceso se caracteriza por el surgimiento de un pliegue del elemento de la finitud sobre el hombre, esto marca la emergencia del mismo, en tanto los seres se dispersan y repliegan en una historia que ya no es el cuadro continuo e infinito de la naturaleza, sino que describe la historicidad misma del hombre y los seres. Con todo, lo que es relevante para nuestro problema es caracterizar la Modernidad desde el punto de vista de la política y las instituciones surgidas con el fin de la "época clásica".

Tal como plantea Foucault (2014), la fractura es efecto del fin del viejo problema del Príncipe/Soberano, que encuentra un ejemplar desarrollo en la obra de Maquiavelo: pensamiento imbuido en la episteme clásica y sus tecnologías de poder cuyo problema es la riqueza y su elevación al infinito, el desarrollo de mecanismos que permitan la conservación y extracción de tierras y súbditos, y la conservación del principado. Sin embargo, el siglo XVIII, marcado por la gesta napoleónica, verá surgir en la ciencia del Estado lo que altera radicalmente el problema del Príncipe/Soberano y el desarrollo de los saberes de la política.

La ciencia del Estado como saber, dice relación con el surgimiento del problema de la Razón de Estado (Foucault, 2014), racionalidad política que recaerá enteramente sobre la figura de la población/público. Dicha racionalidad tiene por objeto el incremento de la salud y felicidad del Estado, lo cual se configura como un fin en sí mismo. Así, para que el Estado posea una buena salud y se mantenga en el tiempo en un estado de gracia, debe reglamentar, normalizar y distribuir correctamente la población desde el punto de vista vital y moral, de modo tal de constituir una "fuerza productiva cuyo efecto deba ser superior a la suma de las fuerzas elementales que la componen" (Foucault, 2018; 90). Esto dice relación con la necesidad del saber 
disciplinar, que se desarrolla a la par de la época de las Luces, de reconducir la finitud del hombre en post de multiplicar sus fuerzas que individualmente son limitadas. Esto abre un problema que será patente a la hora de pensar la democracia representativa que se masifica a lo largo del siglo XIX y XX en Occidente.

Con el desbloqueo que representa la razón de Estado respecto al problema de la Soberanía, surge la figura de la población/público como sujeto e inmediatamente objeto del desarrollo de un conjunto de saberes y tecnologías de poder encaminadas a su abordaje (hospitales, cárceles, talleres, escuelas). Al mismo tiempo que surge la figura de la población: el saber del contractualismo efectuará un "desbloqueo político", en tanto el cuerpo del soberano se desperdigada en el cuerpo político de cada individuo. En este sentido "los dos cuerpos del Rey" de Kantorowicz (2012), es distribuido en el cuerpo de cada átomo social que es el individuo (dispersión) y cuyo efecto debe ser superior a la suma de los elementos que la componen (repliegue del elemento de la finitud). El cuerpo finito del hombre individuo, su vida desde el punto de vista de la población y su cuerpo regio, en tanto ciudadano desde el punto de vista de la representación política. Esto genera una aporía irreductible, entre autonomía y seguridad, entre libertad y conducción (Esposito, 2009).

El desarrollo del liberalismo, como saber y tecnología, desbloquea radicalmente la figura de la población/público. Según Foucault (2014), a medida que el nuevo saber de la economía política se fue desarrollando, puso cada vez más límites internos a la intervención gubernamental del Estado, la cual se ejercía por medio de regulaciones y decretos a la figura de la población. De tal manera, se articularon dos vectores para resolver dicho problema, el primero correspondiente a la solución revolucionaria o rousseauniana (Foucault, 2012), la cual consistió en retrotraerse al derecho natural de la época clásica, pero ahora en una episteme completamente ajena, en tanto la población al mismo tiempo pasa a ser el soberano desperdigado en átomos sociales que son los individuos finitos, con lo cual deviene sujeto/objeto de derecho, tal como en la episteme moderna el hombre pasa a ser sujeto/objeto de interrogación (Foucault, 1968).

Entonces, todo el problema pasaba por delimitar finamente aquellos derechos que correspondían a cada individuo, cuáles eran las maneras en cómo esos derechos son legítimamente traspasados o cedidos, bajo qué mecanismo y en qué momento, cuáles derechos son suprimibles y bajo qué circunstancia. En síntesis, un conjunto de tecnologías jurídicas que son puestas a disposición de un a priori histórico muy diferente. 
Por otro lado, el segundo conjunto de tecnologías de limitación interna de la razón de Estado que analiza Foucault, son los desarrollados por la teoría política anglófona, la cual buscará un límite, ya no desde el punto de vista del derecho, sino desde el punto de vista de la utilidad. En este sentido, el problema de la política pasará enteramente por la capacidad de los gobernantes de gobernar lo menos posible y sólo cuando sea indispensable, basados en el cálculo de la utilidad de la población. El gobierno está anclado enteramente sobre un paradigma administrativo, en tanto gobernar es agenciar, conducir e intervenir sobre el ambiente en el cual se desenvuelve ese elemento heterogéneo y finito que es la población.

Ambos vectores políticos modernos son el alma de lo que hoy conocemos bajo el nombre de democracia representativa: por un lado un conjunto de tecnologías de poder y el desarrollo de unos saberes jurídicos que intentan delimitar y definir el elemento de la representación política disperso en los individuos desde el punto de vista de la transacción legal/legítima de derechos al cuerpo de representantes y cómo estos, al mismo tiempo, son legítimos en tanto capaces de administrar, conducir y gobernar en pos de la utilidad del cuerpo político que deviene así pueblo y población.

En dicho sentido, vemos cómo se delinea el a priori histórico de la modernidad política que atravesará con mayor intensidad la historia de los países occidentales desde el siglo XIX y el sangriento siglo XX. Tanto socialistas, liberales y corporativistas competirán en éste suelo arqueológico identificando y reivindicados diversos sujetos de derecho más legítimos que las figuras precedentes, así como las formas más racionales de administración. El partido, la vanguardia, el líder o el programa no son sino formas particulares de esta racionalidad que intersecta tecnologías jurídicas y tecnologías de conducción. Aquí el elemento de la población desde el punto de vista del público es fundamental para entender la representación política y la formación de la denominada sociedad civil que se tejió mayormente en los últimos dos siglos de historia occidental.

Tal como lo indica Foucault, si la población es el vector desde el punto de vista de los procesos vitales de los individuos, el público es el vector desde el punto de vista de sus opiniones, intereses y deseos, tanto el derecho a la vida como el derecho a la libertad de expresión son la base de aquello que denominamos como democracia representativa. Dicha articulación es la que permite el nacimiento de la sociedad civil como aquello que aúna tanto derecho como conducción. Una superficie de agarre sobre la cual se articulará todo el entramado 
de las instituciones decimonónicas que buscan representar políticamente la población/público, ahora recortada, rostrificada, objetivada, estratificada, posible de procesar, conducir, estimar e identificar.

Todas estas tecnologías de poder y saberes que aún perviven y que se postula han entrado en una crisis irreversible, en la cual el conjunto de tecnologías que ellas mismas habían aunado en un tiempo, han entrado en dispersión, encapsulándose a la espera de su recodificación o de su obsolescencia. Por lo tanto, a continuación revisamos las estrategias adoptadas por la institucionalidad moderna para gestionar el conflicto, así como también la forma en la cual han fracasado, para derivar de ellas una serie de implicancias analíticas.

\section{Primera Arremetida}

Ante el tipo particular de movilización que ha consistido el 18-O, se desplegaron un conjunto de medidas para gestionar el conflicto que había estallado en Chile. Así es como es posible hablar de dos arremetidas paralelas, pero cuya expresión se acentúa en determinados periodos diferenciables entre sí, a saber: la primera se desarrolla principalmente entre los primeros llamados a evadir el pasaje del transporte público, el 7 de octubre, y la multitudinaria marcha del día 25; mientras que la segunda destaca entre el inicio del proceso de cambio de gabinete, el día 26 del mismo mes, y la firma del Acuerdo por la Paz en adelante. En este apartado nos centramos en el primero de ellos.

La primera arremetida, como veremos más adelante, guarda relación con el despliegue de los mecanismos decimonónicos para aplacar la movilización por medio de la producción de una superficie de agarre del poder, que valide el actuar de las fuerzas de orden público y el aparato jurídico. En tal sentido, se trata de un triple movimiento en donde se despliega un dispositivo policial que se encarga más de producir un ambiente susceptible de delictualidad que a prevenirlo (Foucault, 1991) para, luego incluirlo, categorizar e inscribir sobre esos cuerpos el elemento de la exclusión.

El discurso presidencial transmitido en cadena nacional el 19 de octubre, en donde se declara el Estado de Emergencia para la Provincia de Santiago y las comunas de San Bernardo y Puente Alto, contiene estos tres movimientos. A lo largo del primer párrafo, habla de las manifestaciones ocurridas a la fecha, la calificación de los hechos como una alteración del orden público y un atentado contra las libertades individuales: 
"Los graves y reiterados ataques y atentados contra las estaciones y las instalaciones del Metro de Santiago, contra el orden público y la seguridad ciudadana y contra la propiedad tanto público como privada que hemos conocido en los últimos días de la ciudad de Santiago que han afectado gravemente la libre circulación y la seguridad de los habitantes de la ciudad de Santiago y además han alterado gravemente el orden público (Piñera, 19 de octubre de 2019, párr. 1)”.

Días antes de dichas declaraciones, ya se habían desplegado los efectivos policiales en diversas estaciones del tren subterráneo de la capital, situación que, lejos de disuadir a los manifestantes, hizo proliferar los enfrentamientos y los desmanes. Asimismo, si bien la medida se instala previo a una serie de manifestaciones de diversas índoles, se adelanta a las protestas y saqueos ocurridos en el país cuando ya se encontraban desplegados los efectivos de las fuerzas armadas.

Frente a estos hechos, cabría preguntarse ¿Cómo se produjeron dichos hechos, siendo que tantos efectivos de carabineros fueron desplegados antes del Estado de Emergencia y de fuerzas armadas luego de ello? ¿Es posible atribuirlo a una ineficacia de dichos mecanismos o, quizás, a que fueron sobrepasados por la turba de personas agrupadas en las manifestaciones? Una respuesta se hace patente en las declaraciones de Mario Rozas sobre la sensación de ausencia de fuerzas de orden público en las manifestaciones:

Sobre esta manera de actuar, sostuvo que "estamos en un sistema procesal nuevo, donde el estándar de la prueba tiene una exigencia, por lo tanto, nosotros estamos realizando una persecución penal inteligente, en el sentido de que estamos usando la tecnología para poder ubicar y posteriormente detener a todos estos responsables".

"De repente se aprecia como que hubiera un retardo, pero eso obedece a unas estrategias para poder asegurar la salida de operaciones para tener todas las garantías para poder proceder", agregó (Manriquez, 30 de octubre de 2019, párr. 3-4). 
En dicho sentido, hablamos de una estrategia particular cuyo efecto no es la prevención de un acto, sino producirlo para poder codificarlo como punible e inscribirlo sobre un sujeto particularizable. Siendo así, recordamos la sentencia de Foucault (2018) al indicar que, lejos de leer la producción de un campo de delictualidad como un fracaso, es necesario entenderlo como una positividad de sus efectos y no como una intencionalidad oculta (Salinas y Yuing, 2019). Entonces, no es que se quiera incitar al delito, sino que para poder procesarlo es necesario primeramente que sea delito y tener las evidencias para demostrarlo.

De tal manera, se despliega el primer movimiento, el cual requiere ahora de la identificación de los sujetos particulares sobre los cuales inscribir todos aquellos hechos. Por lo tanto, el discurso presidencial ya citado, prosigue en su cuarto párrafo invocando la necesidad de identificarlos y procesarlos penalmente:

"Adicionalmente, como lo anunciamos el día de hoy hemos invocado la Ley de Seguridad del Estado a través de querellas que persiguen el objetivo de que las personas que han cometido esos gravísimos actos de delincuencia enfrenten a la justicia y asuman sus responsabilidades" (Piñera, 19 de octubre de 2019, párr. 4).

Este movimiento, al igual que el anterior, encuentra un correlato en los elementos ulteriores, pues en el discurso del 7 de noviembre cita al Consejo de Seguridad Nacional [COSENA] y anuncia un conjunto de 10 medidas que tienen como finalidad el control del orden público. Lo que ocurre en este conjunto de situaciones es la construcción de un sujeto que sea la contraparte de un cierto "nosotros", una contraparte in-humana que debe rechazarse y que es susceptible de erradicación para garantizar el bienestar, es decir, se despliega una máquina antropológica tal y como la entiende Agamben (2006):

(...) la máquina antropológica (...) funciona en nuestra cultura. En la medida en que en ella está en juego la producción de lo humano mediante la oposición hombre/animal, humano/in-humano, la máquina funciona necesariamente mediante una exclusión (que es también y siempre ya una 
captura) y una inclusión (que es también y siempre ya una exclusión) (Agamben, 2006; 75).

En dicho sentido, sobre el campo de delictualidad producida se erige un sujeto que es primeramente incluido, pero no sin ser al mismo tiempo excluido. En otras palabras, es "uno de nosotros" pero que a la vez es "otro" cuya presencia debe ser identificable en la forma del "vandalismo", el "antisocial", el "extremista", "lumpen", e incluso calificar las movilizaciones como una "invasión alienígena", tal como lo hizo la Primera Dama. Como tercer momento, se posibilita el despliegue de una metonimia de orden biológico sobre el campo social, y que entiende que es necesario eliminar aquella "enfermedad" de la sociedad en una suerte de "racismo de Estado" (Foucault, 2001), lo que queda de manifiesto en las palabras del General de Carabineros Enrique Bassaletti al respecto del uso de la escopeta antidisturbios en las manifestaciones:

"Voy a hacer una analogía, que no sé si es tan feliz, pero la voy a hacer igual. Nuestra sociedad podríamos entreparéntesis decir que en estos días en estos treinta y poco días está enferma de una enfermedad grave.

Supongamos que sea un cáncer, ojalá que no lo sea, y que tenga solución, la va a tener. Pero el tratamiento del cáncer se hace con quimioterapia en algunos casos y otros con radioterapia. Cuando se busca solucionar ese problema, en el ejercicio del uso de esas herramientas médicas se matan células buenas y células malas porque es el riesgo que se somete cuando se usan herramientas como las armas de fuego" (Bassaletti, 22 de noviembre de 2019, s/p).

Lo que queda patente no es solamente la clara referencia a la retórica decimonónica del "cáncer marxista" presente en el despliegue de las fuerzas de orden público, sino por sobre todo la práctica del dispositivo policial, la cual debe desplegar como instrumento: "todo lo que sea necesario y suficiente para que la actividad del hombre alcance una integración efectiva al Estado, a sus fuerzas, al desarrollo de éstas, y deberá procurar que el Estado, a cambio, pueda 
estimular, determinar, orientar esa actividad de una manera eficaz y útil para sí mismo" (Foucault, 2014; 370).

Así, en torno a su labor se articulan cuatro temáticas, a saber: condiciones de vida para subsistir, salud e higiene, producción y circulación de mercancías. En suma, garantizar "la coexistencia de los hombres entre sí" (Foucault, 2014; 375), lo cual no implica que su ejercicio se realice al alero de la justicia, más bien lo realiza en torno a una "actuación directa del monarca sobre sobre sus súbditos (...). En otras palabras, la policía es la gubernamentalidad directa del soberano como tal. Digamos además que la policía es el golpe de Estado permanente" (Foucault, 2014; 388).

En este territorio hablamos de un momento de violencia en donde la ley es suspendida, pero que al mismo tiempo es condición de posibilidad para el resguardo de la ley (Agamben, 2005) y que es leída en términos de un mandato que no pasa por la deliberación del burócrata que es puesto en la posición de ejecutor (Agamben, 1998).

Así queda patente en el tercer párrafo del discurso presidencial del 19 de octubre, ya no solo como la disposición de un campo y la constitución del "otro" como in-humano, sino como la imperiosa necesidad de erradicarlo del campo social como estrategia para garantizar los derechos y libertades individuales democráticos:

"El objetivo de este Estado de Emergencia es muy simple, pero muy profundo: asegurar el orden público, asegurar la tranquilidad de los habitantes de la ciudad de Santiago, proteger los bienes tanto públicos como privados y, por sobre todo, garantizar los derechos de todos y cada uno de nuestros compatriotas que se han visto seriamente conculcados por la acción de verdaderos delincuentes que no respetan a nada ni a nadie, que están dispuesto a destruir una institución tan útil y necesaria como es el Metro, y que además no respetan los derechos ni las libertades de sus compatriotas" (Piñera, 19 de octubre de 2019, párr. 3).

No obstante, esta posición no solamente es ocupada por agentes del Estado, sino también es investida en una serie de sujetos que comienzan a velar por el cumplimiento del derecho regular por medio de su suspensión excepcional. Así, desde el día 20 de octubre, comienzan a 
proliferar las agrupaciones de vecinos que, a pesar del toque de queda, salen durante las noches a resguardar sus barrios que se identifican por vestir un chaleco reflectante y se denominan como "chalecos amarillos". Lo que, además, encuentra un correlato en una serie de sujetos que comienzan a portar armas de fuego para enfrentarse a los manifestantes.

Estos tres movimientos, en su articulación y operatoria tributaria los unos de los otros, se corresponden con la estrategia por excelencia de la democracia representativa para aplacar las movilizaciones por medio de los dispositivos de seguridad. Sin embargo, en esta ocasión, su actuar se ve plenamente deslegitimado y en profunda crisis, lo cual se hace patente en dos situaciones particulares, a saber: (1) la valoración del encapuchado en las marchas y (2) el rechazo y crítica tanto al actuar como a los protocolos y procedimientos de las fuerzas de orden público.

En cuanto a los encapuchados, se vuelve característica la denominación de "primera línea" como sintagma que reemplaza a las anteriormente empleadas por la opinión pública en términos de "antisocial" o "delincuente". Asimismo, esta novedosa nomenclatura refiere a su actuar como aquellos que detienen, o al menos entorpecen, el actuar de Fuerzas Especiales para que las marchas se desarrollen sin problemas.

Siguiendo esta línea, se instalan una serie de figuras icónicas de las movilizaciones que se caracterizan por ser propiamente inidentificables o no que no son posibles de reducir a un sujeto en particular. Así es como destacan tanto el renacimiento del "Negro Matapacos" y de "Pareman". El primero como un animal, un perro mestizo que solía participar de las movilizaciones estudiantiles y los enfrentamientos realizados en el frontis de la Universidad de Santiago de Chile hasta el día de su muerte. Mientras que, el segundo, como un joven encapuchado que sostiene un "disco pare" a modo de escudo y que fue fotografiado durante las manifestaciones.

Ambos personajes, como figuras de las movilizaciones, carecen de rostro y no remiten en realidad a ningún sujeto en particular: son nómades, y en ellas confluyen una serie de individuos dispares y cuyas demandas no son posibles de ser resueltas en un aparato parlamentario. Siguiendo las palabras de Mauro Salazar (21 de noviembre de 2019), es una fuerza indomable, innombrable e indecible; un movimiento vertiginoso y diluviano, sin mito, ni promesa, pero que goza de una inaprensible fuerza de legitimidad. 
Por otro lado, se evidenció una profunda crítica a los procedimientos desplegados por las fuerzas de orden público. Destaca la producción de una serie de gestos que son la respuesta al estallido de globos oculares producto del impacto de perdigones y al uso de la "escopeta antidisturbios". En tal sentido, se vuelve recurrente el acto de taparse un ojo en señal de desprecio y denuncia del actuar de Fuerzas Especiales.

Asimismo, son las propias instituciones de la modernidad, en un plano tanto nacional como internacional, las que comienzan a emitir una serie de informes que rechazan las estrategias de disuasión de las fuerzas de orden público en el país, tales como fueron los esfuerzos del Instituto Nacional de Derechos Humanos, Amnistía Internacional y Human Rights Watch.

En dicho sentido, la primera arremetida encuentra su rotundo fracaso en un primer rasgo del 18-O como un conjunto de movilizaciones que no pueden ser aprehendidas en términos de un "órgano patológico" de lo social y, por lo tanto, las tecnologías securitarias para su regulación y "normalización" carecen de una legitimidad interna, pues no responden a su lógica. En otras palabras, hablamos de una serie de manifestaciones que, en su acople y resonancia, operan por medio de una derogación de las formas sociales tal y como son conocidas en la actualidad, y no como un evento predecible, calculable y gestionable dentro de la episteme moderna.

\section{Segunda Arremetida}

La imposibilidad de gestionar el conflicto de la primera arremetida es volcada sobre una segunda estrategia política, cuya operatoria se desliga de las movilizaciones y se vuelca sobre las figuras del gobierno y las clases políticas como vector de solución. Dicho de otra forma, se asume que el conflicto no es sino una problemática de representatividad, es decir, la imposibilidad del sistema político de procesar y aunar la representación política dispersa en la figura del pueblo/población, en las instituciones diseñadas para estos fines. De este modo, la intelección del 18-O resulta sintomática del régimen de veridicción de la modernidad desde el cual las elites han intentado establecer cierto número de certezas cognitivas a propósito del "estallido social". Es importante destacar que esta segunda arremetida no implica un cese de la primera, sino un foco paralelo de acción.

Este desplazamiento queda patente en el discurso presidencial que nombra a los nuevos ministros de Estado, pues, asumiendo que los mecanismos modernos no han dado lugar a una resolución, se declara que es el propio gobierno el que no ha sabido llegar a acuerdos y es 
menester que se adapte a los nuevos tiempos: "Chile no es el mismo que el que teníamos hace un par de semanas; Chile cambió y el Gobierno también tiene que cambiar para enfrentar estos nuevos desafíos y estos nuevos tiempos" (Piñera, 28 de octubre de 2019, párr. 20).

De tal manera, el actuar de la clase política en este punto se encuentra bajo la idea de que las movilizaciones han adquirido un matiz en el cual todos los elementos vinculados a las estrategias de la primera arremetida producen una re-dirección inmediata a ciertas figuras, siendo el principal afectado de esta dinámica el, en ese entonces, Ministro del Interior Andrés Chadwick. Siguiendo a Deleuze y Guattari (1994), se estaría frente a una rostrificación del problema institucional que no se corresponde con la cara como imagen o como modelo, sino como una "sobrecodificación para todas las partes descodificadas" (Deleuze y Guattari, 1994; 176). Ello implica que una serie de elementos tales como las denuncias de violaciones a los derechos humanos y la brutalidad policial son encarnadas en esta figura del gobierno, a la cual se le asigna un rostro que sobrecodifica en él todo los elementos caóticos. Con esto se busca hacer visible y representable a la mirada un sujeto de la culpa.

No obstante, al corto plazo, esta opción no genera un decrecimiento de las manifestaciones y el esquema de lectura cambia luego del Paro Nacional del 12 de noviembre, día en el cual el presidente vuelve a dar un discurso en cadena nacional refiriéndose ahora sobre nuevos tres focos sobre los cuales pretende encontrar un punto de sobrecodificación de la crisis:

"Quiero decirlo con toda claridad, desde el primer día nuestro Gobierno ha hecho todos los esfuerzos posibles por escuchar a la gente con atención, con humildad, con comprensión y también por buscar acuerdos con todos los sectores, con buena voluntad, con capacidad de diálogo y con flexibilidad, porque estamos absolutamente convencidos que debemos y tenemos que impulsar todos juntos estos tres grandes Acuerdos; el Acuerdo por la Paz, el Acuerdo por la Justicia y el Acuerdo por una nueva Constitución" (Piñera, 12 de noviembre de 2019, párr. 11).

La convocatoria a tres acuerdos pretende haber encontrado los puntos de conflicto en los cuales es posible leer el 18-O, a saber: un acuerdo por la paz que busca condenar las violencias y a quienes la impulsan, un acuerdo de justicia que busca avalar la agenda social del 22 de octubre 
como forma de reducir desigualdades, y un acuerdo por una nueva constitución que pretende modificar la carta fundamental sin alterar la institucionalidad basada en la representación política que, pareciera, se encuentra en una crisis irreconciliable.

Así es como, la madrugada del 15 de noviembre, la clase política firma el Acuerdo por la Paz Social y la Nueva Constitución, en donde se logra materializar en su máxima expresión la segunda arremetida. El primer punto de dicho documento señala enfáticamente que son los partidos políticos los que comprometen a garantizar la paz y el orden público, así como también la institucionalidad democrática vigente. Situación que no hace sino re-editar una (no tan) antigua sentencia sobre Chile:

"Las promesas de movilización chocaron contra el realismo de la dirigencia política que se sentía al borde de alcanzar el gobierno, chocaron contra la obstinación de un gobierno que continuó usando las armas de la represión y la amenaza de la involución. Pero, además, chocaron contra un deseo profundo de la multitud, que no alcanzó estatura discursiva pero que no por ello era menor vivo: el deseo de normalización. Ese deseo de no tener que continuar desempeñando papeles heroicos, de que la política perdiera su "insoportable gravedad", fue una de las claves secretas de la coyuntura postplebiscitaria" (Moulian, 1997; 354).

La segunda arremetida con sus negociaciones, sus llamados a la democracia, nos retrotraen al pasado reciente del país. Hablamos de un revival de la Democracia Pactada, como un agónico cuerpo que se niega a morir y que ve en antiguas estrategias, ya empleadas para un vuelco institucional del conflicto, una forma de superar las movilizaciones desembocadas por el 18-O. De tal manera, no es extraño que a partir del día de la firma comenzara una estratagema política paralela que buscaba blanquear al país.

En la mañana del mismo día, Chile despierta ya no solo con un acuerdo, sino con la imagen de Plaza Italia, en tanto centro neurálgico de las manifestaciones, cubierta por un lienzo blanco que, no conforme con ocultar bajo si las marcas de casi un mes ininterrumpido de concentraciones, protestas y enfrentamientos, erigía sobre la estatua de Manuel Baquedano la 
consigna: "Paz". Una imagen de similares características al iceberg que ocupaba un lugar en el Pabellón de Chile de la Expo 1992 de Sevilla, sobre el cual Moulian (1997) escribe:

"El iceberg fue la escultura de nuestra metamorfosis. El iceberg estableció ante los ojos del mundo la transparencia del Chile Actual. Todas las huellas de sangre, de existir, estaban cristalizadas en un azul profundo. Los tormentos, de existir, eran ahora las vetas blancas del hielo" (Moulian, 1997; $34)$.

Tal como lo analiza Moulian el iceberg no solamente fue señal de una serie de avances tecnológicos que permitieron mostrar la potencialidad del Chile post-Pinochet como modernizado, sino que también aquella mole blanca venía a tapar la historia, a mostrarse como una página en blanco sobre la cual comenzar a escribir la historia del progreso que marcaría al país. Por lo cual, vemos en el conocido "Lienzo de la Paz" la misma pretensión blanqueadora del conflicto, el suplicio y la movilización, como una figura de resolución acordada por la institucionalidad moderna, una especie de esfuerzo meteórico por restituir la representación pérdida y dispersada por el movimiento diluviano (Deleuze, 2005) que amenaza siempre con consumir desde el fondo los cimientos del orden newtoniano de la modernidad (Foucault, 1968).

No obstante, esta pretensión de blanquear al país se vio plenamente "manchada" del color rojo del que buscaba escapar. Durante la tarde del mismo día, en las manifestaciones que no cesaron, un joven muere en Plaza Italia producto de un edema pulmonar causado por el agua proveniente del carro lanza agua empleado por las Fuerzas Especiales de carabineros para la disuasión de los manifestantes. Rompiéndose, así, el primer punto del Acuerdo por la Paz Social y la Nueva Constitución.

El Acuerdo por la Paz Social y la Nueva Constitución, presenta además, un elemento a todas luces sintomático de la imperiosa necesidad de restituir en las instituciones políticas el elemento de la representación de la sociedad, en tanto el acuerdo entre los partidos políticos realiza un movimiento que mimetiza en el parlamento el conflicto callejero. Esto debido que el acuerdo se presenta y representa un armisticio entre partes que miméticamente representan a las partes en conflicto en las calles de Chile. Los parlamentarios firman un documento en el que acuerdan terminar con las hostilidades, sin embargo este gesto resulta sólo paródico. 
Siendo así, en dicho documento operan dos mecanismos que cooptan las potencialidades para limitarlas a los marcos preestablecidos por la esquemática de la democracia representativa. Por un lado, la mimesis que realiza el poder político institucional del conflicto social. Por otro, lo encuadra dentro del sistema electoral vigente que garantiza la "partidocrácia", en una especie de sistema de cuotas que excluye la posibilidad de que independientes fuera de ellos puedan ser electos.

Entonces, hablamos de un pacto político-oligárquico que "consiste en la demostración de que se podía pasar de la desconfianza y de la odiosidad del periodo de la lucha, al acuerdo perfecto de la transición" (Moulian, 1997; 38). Dicho de otra forma, no solamente nos encontramos ante el blanqueamiento de un Chile Porvenir, sino también en una promesa de cambio que, a la larga, re-configura el escenario para hacer que nada cambie. Un retorno a la racionalidad moderna de la institucionalidad que, acallando las voces del sufrimiento y la lucha (Richard, 2010), se repliega sobre sí misma para tratar de legitimarse en una crítica interna que aspira a reformularse para adaptarse.

No obstante, esta segunda arremetida encuentra un profundo rechazo por parte de las manifestaciones, las cuales continúan en el país y quedan graficadas en una icónica "funa” a la ex candidata a la presidencia por el Frente Amplio, Beatriz Sánchez. Sin embargo, también en cómo el Acuerdo por la Paz y la Nueva Constitución pasa a ser denominado por Pamela Jiles, parlamentaria del Partido Humanista, quien lo designa como una "cocina" para hacer referencia su constitución como un acuerdo oligárquico; crítica que paulatinamente se extiende y pasa a conformar parte de los enclaves de las movilizaciones.

En suma, la segunda arremetida busca hacer una transubstanciación de las protestas hacia una forma de conflicto interno del propio aparataje institucional de la modernidad, considerando que en este último radican las causas últimas de las movilizaciones y que, por lo tanto, es menester una actualización como vía de escape. Siendo así, resalta el segundo rasgo del 18-O ya presente en las conclusiones de la primera arremetida, es decir, el mismo fin de la democracia representativa en una exigencia por otras formas de actuación que, hasta la fecha, no adquieren una forma estabilizada. 


\section{Conclusiones: Devenir Imperceptible}

A lo largo del presente artículo, hemos realizado un análisis coyuntural del 18-O con la doble finalidad de otorgar un panorama político del contexto nacional y de poder abstraer la disyunción existente entre el esquema decimonónico de la democracia representativa y una conformación social que escapa continuamente. En tal sentido, abordamos el suelo arqueológico desde donde se erige la racionalidad moderna, así como también las formas en las cuales las protestan no se han dejado reducir a dicha esquemática. Ahora, finalmente, es momento de revisar las últimas consecuencias de dicho trabajo a la luz de nuestra hipótesis central.

La modernidad, con sus figuras y figuraciones del Hombre, es acompañada por un creciente proceso de desterritorialiación y decodificación (Deleuze, 2005). La modernidad como dispersión y pliegue del elemento de la representación, es por sí misma víctima de su propia tragicidad. Una agonística interna que ha agotado su poder de movilización del campo de lo social por la senda de sus órdenes institucionales, al punto de que éstas misma instituciones se encuentran extenuadas en procesos de creciente financiarización de la economía, digitalización de los espacios laborales, proliferación de procesos de subjetivación que ya no pasan por las instituciones tradicionales, al punto de minar toda legitimidad.

En este sentido, el 18-O comparte con el capitalismo contemporáneo el carácter rizomático y diluviano, en tanto que barre con toda forma de territorialidad y codificación de los cuerpos a espacios y áreas asignadas (Lash y Urry, 1998). No obstante, el 18-O es propiamente un "flujo revolucionario" (Deleuze, 2005) o un "devenir imperceptible (Deleuze y Guattari, 1994), puesto que, a diferencia del capitalismo, este no busca restituir una axiomática, establecer un umbral antes del límite.

Por lo tanto, no se rostrifica, no conforma vanguardias, no busca establecer el proceso mimético de la representación por medio de su institucionalización. Siendo así, sería inútil leer las movilizaciones del último tiempo como una continuación del 2011 por otros medios. Ello debido a que el 2011 estudiantil fue un proceso de protestas capitalizada por partidos, dirigencias y vanguardias que en observancia a la episteme moderna, buscaban representar el descontento disperso y descodificado al interior de las instituciones, es decir, establecer dicho umbral.

En contraste, el 18-O puede ser conceptualizado como una revuelta (Arditi, 2013) o como un proceso de destitución (Agamben, 2017), en tanto no renueva el ciclo mítico de la modernidad, entre dispersión y pliegue. La protesta social ha adquirido una singular corporalidad 
inorgánica, ya que posee una corporalidad sin orden posible. Así, se extendió por la ciudad en un desborde de la fuerza policial constante, es por esto que la "primera arremetida" fracasa tan estrepitosamente: ante la pregunta ¿quién incendió la ciudad? no hay sujeto, ni rostro identificable.

Aclaremos de inmediato: no nos referimos aquí a una especie de inmaterialidad en la acción, sino que más bien a una mutación en los regímenes de veridicción o la episteme, es decir, una modificación en el régimen de lo enunciable y lo visible (Deleuze, 1987; 2018). En este encuadre, no hay un "primer sujeto" sobre el cual adjudicar la acción gatilladora, sino un conjunto de resonancias en las cuales no es posible identificar un "origen", no importando si éste existiese o no.

El orden del neoliberalismo hacendal y mercantilista chileno, que tan brillantemente describieron en sus trabajos Moulian (1997) y Salazar (2009; 1989), ha entrado en una crisis que a todas luces resulta irreversible, no porque que la revuelta haya derribado el gobierno de turno, sino por una razón más simple: la revuelta ha mostrado la grieta que atraviesa el desarrollo político chileno desde hace ya al menos 40 años, es decir, una oligarquía bursátil y especulativa pero al mismo tiempo hacendal y rentista, anclada en una modernidad que ha entrado en crisis y que ya no es sostenible en el tiempo.

La revuelta ha mostrado la fragilidad de dicho equilibrio y las instituciones se han autorecetado un conjunto de medidas que han resultado obsoletas ante el nomadismo de una movilización social que se extiende y contrae, acelera su ritmo y frena, mientras se desparrama por la ciudad. Estamos ante una revuelta post-representacional, que desconfía de toda mimética institucional y la muestra como paródica (Haraway, 1999).

Tal como se ha visto, la democracia representativa, en su despliegue de libertades individuales, opinión pública y resguardo policial de dichas garantías, se encuentra frente a una forma de organización social acéfala, es decir, que no se corresponde con líderes ni vanguardias y cuyo despliegue recuerda más a un cúmulo de acontecimientos que actúan en resonancia los unos con los otros. En tal sentido, no es anómalo, ni anímica, ni irracional, pues se encuentra por fuera operando en una racionalidad completamente diferente e irreductible.

Por lo tanto, la crisis que hemos descrito se plantea como una profunda disyunción, una confrontación entre dos epistemes que tiene como efecto el fin de la democracia tal y como la hemos conocido hasta la actualidad. 
Asimismo, sus arremetidas y mecanismos particulares de gestión del conflicto se encuentran mucho más cercanos al fascismo, pero esto no porque la democracia sea lo mismo que el fascismo, sino que tanto la democracia representativa como el fascismo comparte el mismo suelo epistémico, con esto la interrogación de Esposito (2009), Brossat (2008) o Agamben (2013) a la democracia representativa y su borrosos límite con la experiencia totalitaria toma todo el sentido, si se aborda desde el punto de vista de la episteme que comparten: la moderna. Es Walter Benjamin, quien roza este momento de verdad, al señalar que:

"El fascismo intenta organizar a las masas proletarias que se han generado recientemente, pero sin tocar las relaciones de propiedad hacia cuya eliminación ellas tienden. Tiene puesta su meta en lograr que las masas alcancen su expresión (pero de ningún modo, por supuesto, su derecho). Las masas tienen un derecho a la transformación de propiedad; el fascismo intenta darles expresión que consiste en la conservación de esas relaciones. Es por ello que, el fascismo se dirige hacia un estetización de la vida política" (Benjamin, 2003; 96)

Vemos, entonces, cómo la episteme moderna y cincelada en Chile por la transitología y los transitólogos luego de la Dictadura Militar, no solo no logra aplacar y procesar tanto intelectual como institucionalmente la revuelta, lo que la fuerza a la deriva que se ha denominado como la primera arremetida, una máquina de muerte que busca producir una superficie de agarre sobre la cual aferrarse a mantener un orden y una segunda que pretende dar una respuesta erigiendo grandes pactos, figuras mesiánicas de salvación y miméticas de la representación.

En suma, nos encontramos ante un acontecimiento, una grieta profunda en las formas del saber de la política conocidas hasta el momento, que tiene la potencialidad de abrirse a lo múltiple. Sin embargo, no es un motivo para caer en la optimista y positiva lectura marxista de la lucha de clases hacia la liberación. Todo lo contrario, es menester prestar atención a los mecanismos empleados en su resolución, los nuevos saberes que proliferaron en este nevoso suelo arqueológico y los mecanismos de poder que pulularán en él.

Recordando a Deleuze (2006), el momento actual no da "lugar para el temor ni para la esperanza, sólo cabe buscar nuevas armas". 


\section{Bibliografía}

Acuerdo por la Paz Social y la Nueva Constitución. (15 de noviembre de 2019). Santiago, Chile. Recuperadode:https://www.senado.cl/senado/site/mm/20191114/asocfile/20191114134609/pdf_a cuerdo_por_la_paz_social_y_la_nueva_constitucion.pdf

Agamben, Giorgio. (1998).¿Qué es un campo?. Nombres, (10), 91-96.

Agamben, Giorgio. (2005). Estado de excepción. Homo sacer, II, I. Buenos Aires: Adriana Hidalgo.

Agamben, Giorgio (2006). Lo abierto. El hombre y el animal. Buenos Aires: Adriana Hidalgo.

Agamben, Giorgio (2013). Homo sacer. El poder soberano y la vida. Valencia: Pre-textos.

Agamben, Giorgio (2017). El uso de los cuerpos: homo sacer IV, 2. Buenos Aires: Adriana Hidalgo.

Arditi, Benjamín. (2013). "Las insurgencias No Tienen un Plan -Ellas Son el Plan: Performativos Políticos y Mediadores Evanescentes”. Revista Sul-Americana de Ciência Política, 1(2), 1-18.

Bassaletti, Enrique. (22 de noviembre de 2019). General de Carabineros compara represión con quimioterapia: "Se matan células malas y células buenas". El Desconcierto [En línea]. Recuperado de: https://www.eldesconcierto.cl/2019/11/22/general-de-carabineros-compararepresion-con-quimioterapia-se-matan-celulas-malas-y-celulas-buenas/.

Benjamin, Walter. (2003). La obra de arte en la época de su reproductibilidad técnica. México D.F.: Itaca.

Brossat, Alain. (2008). La democracia inmunitaria. Santiago; Palinodia.

Deleuze, Gilles. (1987). Foucault. Barcelona: Paidós.

Deleuze, Gilles. (2005). Derrames. Entre el capitalismo y la esquizofrenia. Buenos Aires: Cactus.

Deleuze, G. (2006). Post-scriptum sobre las sociedades de control. Polis [En línea], 13. Recuperado de: https://journals.openedition.org/polis/5509.

Deleuze, Gilles. (2018). El saber: curso sobre Foucault. Buenos Aires: Cactus.

Deleuze, Gilles., y Guattari, Felix. (1994). Mil mesetas: Capitalismo y esquizofrenia. Valencia: Pre-textos.

Foucault, Michel. (1968). Las palabras y las cosas, una arqueología de las ciencias humanas. Buenos Aires: Siglo XXI editores. 
Foucault, Michel. (1979). La arqueología del saber. México D. F.: Siglo XXI editores.

Foucault, Michel. (1991). Nuevo orden interior y control social. En, Saber y Verdad (pp. 163166). Madrid: Las Ediciones de la Piqueta.

Foucault, Michel. (2012). Nacimiento de la Biopolítica. Curso en el Collège de France (19781979). Buenos Aires: Fondo de Cultura Económica.

Foucault, Michel. (2014). Seguridad, Territorio y Población. Curso en el Collège de France (1977-1978). Buenos Aires: Fondo de Cultura Económica.

Foucault, Michel. (2018). Vigilar y castigar: nacimiento de la prisión. Buenos Aires: Siglo XXI editores.

Esposito, Roberto. (2009). Inmunitas. Protección y negación de la vida. Buenos Aires: Amorrortu.

Fleet, Nicolás. (2011). "Movimiento estudiantil y transformaciones sociales en chile: una perspectiva sociológica”. Polis [En línea], 30. Recuperado de:

http://journals.openedition.org/polis/2152.

Garcés, Mario. (2015). El movimiento de pobladores durante la Unidad Popular, 1970-1973. Atenea, (512), 33-47.

Haraway, Donna. (1999). "La promesa de los monstruos: una política regeneradora para otros inapropiados/bles”. Política y Sociedad, 30, 121-163.

Lash, Scott., y Urry, John. (1998). Economías de signos y espacios. Sobre capitalismo de la posorganización. Buenos Aires: Amorrortu.

Manriquez, Yem. (30 de octubre de 2019). Rozas sobre "ausencia" de Carabineros en saqueos: Es una estrategia de persecución penal inteligente. $A D N$ [En línea]. Recuperado de:

https://www.adnradio.cl/noticias/nacional/rozas-sobre-ausencia-de-carabineros-en-saqueos-esuna-estrategia-de-persecucion-penal-inteligente/20191030/nota/3973120.aspx.

Moulian, Tomás. (1987). Chile Actual: anatomía de un mito. Santiago: LOM.

Núñez, Daniel. (2012). "Proyecciones políticas del movimiento social por la educación en Chile". Osal, 13(31), 61-70.

Ortega, Luis. (2008). "La radicalización de los socialistas en Chile en la década de 1960". Universum, 23(2), 152-164.

Piñera, Sebastián. (19 de octubre de 2019). Presidente Piñera decreta Estado de Emergencia en las Provincias de Santiago y Chacabuco y en las comunas de San Bernardo y Puente Alto para normalizar el orden público [En línea]. Recuperado de:

https://prensa.presidencia.cl/discurso.aspx?id=103651. 
Piñera, Sebastián. (28 de octubre de 2019). Presidente Piñera nombra nuevos ministros [En línea]. Recuperado de: https://prensa.presidencia.cl/discurso.aspx?id=133961.

Piñera, Sebastián. (12 de noviembre de 2019). Presidente Piñera realiza llamado a acuerdos por la paz, justicia social y nueva Constitución [En línea]. Recuperado de:

https://prensa.presidencia.cl/discurso.aspx?id=134376.

Richard, Nelly. (2010). Crítica de la memoria. Santiago: Ediciones Universidad Diego Portales.

Rifo, Mauricio. (2013). "Movimiento estudiantil, sistema educativo y crisis política actual en Chile”. Polis [En línea], 36. Recuperado de: http://journals.openedition.org/polis/9469.

Salazar, Gabriel. (1989). Labradores, peones y proletarios. Formación y crisis de la sociedad popular chilena del siglo XIX. Santiago: Ediciones Sur.

Salazar, Gabriel. (2007). Mercaderes, empresarios y capitalistas (Chile, siglo XIX). Santiago: Editorial Sudamericana.

Salazar, Mauro. (21 de noviembre de 2019). Destitución, primera línea y potencia plebeya. El Desconcierto.cl [En línea]. Recuperado de:

https://www.eldesconcierto.c1/2019/11/21/destitucion-primera-linea-y-potencia-plebeya/

Salinas, Adán., y Yuing, Tuillang. (2019). "Focos y desenfoques en la analítica del poder: apuntes metodológicos". Revista de Filosofía Aurora, 31(51), 74-93.

Toro, Pablo. (2015). «Tiempos tristes»: notas sobre movimiento estudiantil, comunidad y emociones en la Universidad de Chile ante la dictadura de Pinochet (1974-1986). Espacio, Tiempo y Educación, 2(2).

Valderrama, Miguel. (2016). Coloquio sobre Gramsci. Santiago: Palinodia. 